\title{
Role of Lysyl Oxidases in Neointima Development in Vascular Allografts
}

\author{
M. Rodríguez ${ }^{b} \quad$ G. Pascuala ${ }^{a} \quad$ A. Cifuentes ${ }^{a} \quad$ B. Perez-Köhler ${ }^{a} \quad$ J.M. Bellón ${ }^{b}$ \\ J. Buján ${ }^{a}$ \\ Departments of a Medical Specialities and ${ }^{\text {b}}$ Surgery, Networking Research Center on Bioengineering, \\ Biomaterials and Nanomedicine (CIBER-BBN), Faculty of Medicine, Alcalá University, Alcalá de Henares, Spain
}

\section{Key Words}

Vascular allografts $\cdot$ Intimal hyperplasia $\cdot$ Lysyl oxidases $\cdot$

Extracellular matrix $\cdot$ Lysyl oxidase inhibitors

\begin{abstract}
Background: Extracellular matrix deposition is the main factor inducing stenotic lesions in arterial grafts. Lysyl oxidases (LOX) play a key role in stabilizing collagen and elastin. Objective: To examine the repair response to arterial allografts in terms of LOX expression and collagen/elastin deposition using LOX inhibitors. Methods: Lewis/Fisher-344 rats were used as donors/recipients. Donor segments were grafted to the right iliac artery of recipients and retrieved 14/30 (short-term) or 90/180 days (long-term) after surgery. One group of animals was injected with a potent irreversible LOX inhibitor daily for 30 days. Results: Intimal hyperplasia increased in thickness until 90/180 days postsurgery. Elastin showed great expression in the neointima at 14/30 days and in the media at 90/180 days. LOX/LOXL1 were similarly expressed in the arterial wall during the first month. In the long term, their overexpression was confined to neointimal layers. At 14 days, collagen types I/III were identified in the grafts. The neointima acquired collagen I over time. In the
\end{abstract}

group of animal treated with the LOX inhibitor, intimal hyperplasia was significantly inhibited. Conclusion: LOX were overexpressed in late stages of intimal hyperplasia in the allografts. LOX inhibitors prevented the development of the neointimal layer, such that their modulation could reduce the excessive extracellular matrix deposition that leads to stenosis.

Copyright $\odot 2010$ S. Karger AG, Basel

\section{Introduction}

Although the use of the saphenous vein or internal mammary artery as a vessel substitute is the best option, this approach is limited by the necessity of finding a vessel of adequate diameter. The search for vascular substitutes, especially to replace small- and medium-caliber vessels, continues to be the objective of numerous investigations $[1,2]$.

When the arterial wall is injured, intimal hyperplasia causes a change in vascular structure. Intimal hyperplasia, defined as abnormal cell proliferation and migration, leading to extracellular matrix deposition, is identified as stenosis and is attributed to the arterial complications

\section{KARGER}

Fax +41613061234

E-Mail karger@karger.ch

www.karger.com
(C) 2010 S. Karger AG, Basel

1018-1172/11/0481-0043\$38.00/0

Accessible online at:

www.karger.com/jvr
Dr. J. Buján

Department of Medical Specialities

Faculty of Medicine, University of Alcalá

Ctra. Madrid-Barcelona Km 33.6, ES-28871 Madrid (Spain)

Tel. +3491885 4540, Fax +3491885 4885, E-Mail mjulia.bujan@ uah.es 

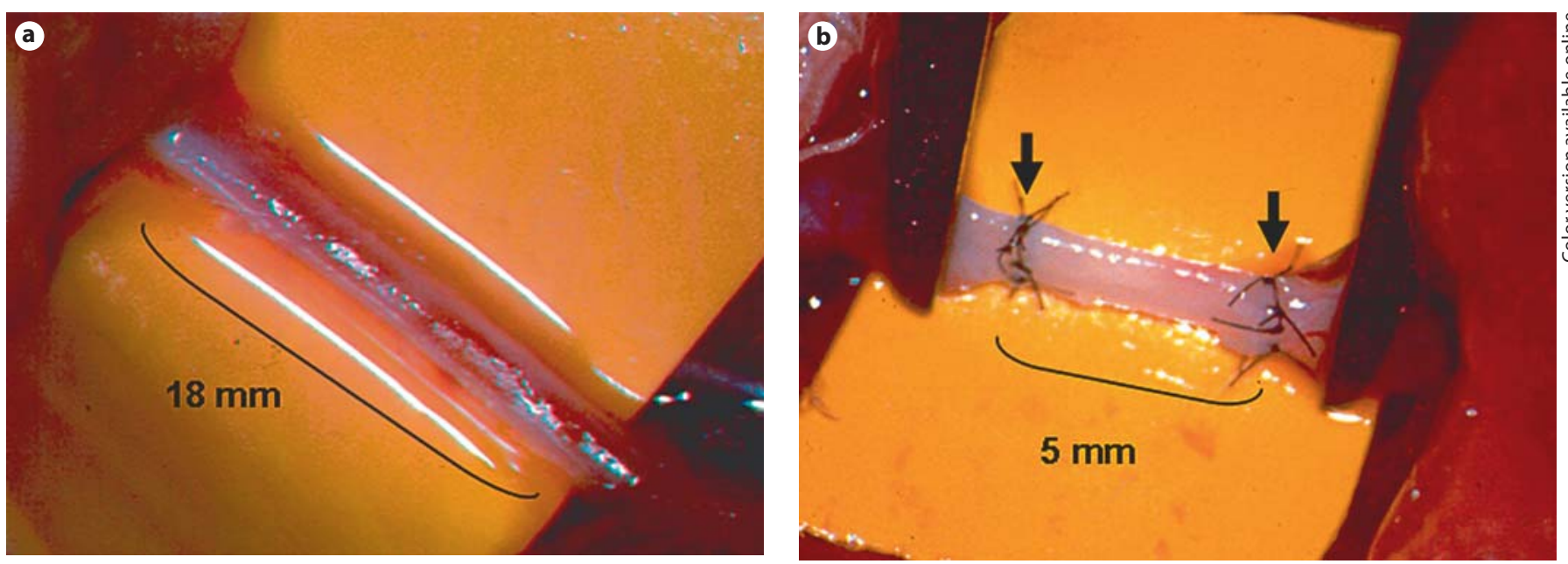

Fig. 1. a Isolating a segment of the right iliac artery into which the allograft will be implanted. b Fresh arterial segment $(5 \mathrm{~mm})$ grafted to the right iliac artery of a recipient animal by end-to-end anastomosis (arrows) using $10 / 0$ suture thread.

that take place between the first 2 months and 2 years after a vascular surgery procedure.

In some cases, hyperplastic growth stabilizes and the luminal area of the vessel remains within a functional range. In other cases, the growth of this neolayer can lead to stenosis, restenosis and/or obstruction of the vessel lumen. Extracellular matrix deposition is the main factor involved in the growth of stenotic and restenotic lesions and lysyl oxidases (LOX) play a key role in stabilizing collagen and elastin.

LOX are enzymes that achieve the cross-linking of fibers during collagen and elastin synthesis by converting lysin and hydrolysin residues to aldehydes. LOX activity modulation induces different effects on the structure and main characteristics of the extracellular matrix. LOX are produced by smooth muscle cells and activated fibroblasts and have been thought to be involved in stabilizing the connective tissue of vessels and maintaining adult tissues. They also play a vital role in extracellular crosslinking and stabilization of collagen and elastin in response to injury in a great variety of tissues [3]. These enzymes are essential for maintaining the characteristics of blood vessels, and its modulated activity has been reported in aneurism and atherosclerosis $[4,5]$. Its downregulation has been related to connective tissue disorders seen in Ehlers-Danlos syndrome, Menkes syndrome and cutis laxa [6-8].

The present descriptive observational study was designed to evaluate the repair response to arterial allografts by analyzing LOX expression in the presence and absence of a LOX inhibitor and correlating this expression with elastin and collagen deposition.

\section{Materials and Methods}

\section{Experimental Animals}

Experimental animals were housed and handled according to the terms and codes of ethics required by Spanish and European legislation (Law 32/2007, Spanish Royal Decree 1201/2005, European Directive 609/86/CEE and European Council ETS 123).

Seventeen female Lewis rats were used as donors and 34 female Fisher-344 rats as recipients to establish the study groups. In all the cases, 4-month-old rats were used. Graft segments were procured from both common iliac arteries of the donor animals. Nongrafted fresh $(\mathrm{n}=5)$ arterial segments served as controls.

Fresh arterial segments $(5 \mathrm{~mm})$ were grafted to the right iliac artery of recipient animals by end-to-end anastomosis using 10/0 suture thread (fig. 1). The animals were not treated with anticoagulants or immunosuppressive drugs pre- or postoperatively. The grafts were recovered at $14(\mathrm{n}=7), 30(\mathrm{n}=7)$ (short-term), 90 $(n=7)$ or $180(n=7)$ days (long-term) after surgery for morphological, morphometric and immunohistochemical analysis.

After grafting, one group of animals $(n=6)$ received injections of $3 \beta$-aminopropionitrile fumarate (BAPN), a potent irreversible LOX inhibitor (-LOX). The BAPN salt (Sigma-Aldrich, USA) was dissolved in $0.9 \% \mathrm{NaCl}$ prior to injection. All 7 rats were given daily intraperitoneal injections of BAPN $182 \mathrm{mg} \mathrm{kg}^{-1}$, equivalent to $100 \mathrm{mg}$ BAPN free base, for 30 days.

Graft specimens for microscopic observation were obtained as follows. After anesthetizing the animal, the blood system was perfused first with saline to eliminate all the blood, and then with a 
mixture $(2: 1)$ of $3 \%$ glutaraldehyde: $1 \%$ paraformaldehyde for $10-$ $15 \mathrm{~min}$ to avoid tissue shrinkage. After perfusion, the grafted segment was extracted, preserving some of the recipient artery at both the distal and proximal ends.

Specimens were fixed with 10\% paraformaldehyde (for morphological analysis) or Bouin's fluid (for immunohistochemistry), embedded in paraffin and cut into $5-\mu \mathrm{m}$ serial sections using a rotating microtome Microm HM-325, and then deparaffinated and rehydrated.

\section{Morphological Analysis}

To examine the morphology of the arterial wall, sections for histology were stained using hematoxylin-eosin, Masson's trichrome (Goldner-Gabe variant) and orcein before observation under a light microscope (Zeiss Axiophot, Jena, Germany).

Morphometry of Intimal Hyperplasia and Tunica Media

The thickness of the neointima and medial layer was determined in histological sections of the grafts stained with hematoxylin-eosin, orcein or Masson's trichrome. Twenty digitalized histological images per animal were obtained using a digital camera fitted to the microscope (Axiocam HR, Zeiss) and analyzed using image analysis software Axiovision AC 4.1.

The thickness of the tunica intima was measured from the vessel lumen to the internal elastic lamina, while the thickness of the media was taken as the distance between the internal elastic lamina and the external elastic lamina (fig. 2b, d, f). Statistical analysis was performed using a nonparametric test. Neointimal and medial thicknesses were compared among the study groups using the Mann-Whitney U test. The level of significance was set at $\mathrm{p}<0.05$.

Immunohistochemical Analysis

Deparaffinated and hydrated sections were equilibrated in PBS buffer ( $\mathrm{pH}$ 7.4). The antibodies used were rabbit polyclonal anti-tropoelastin (1:500), rabbit monoclonal anti-LOXL1 (1:200) and anti-LOX (1:500) antibodies. The antigen-antibody reaction was detected by peroxidase-labeled avidin-biotin procedures. The chromogenic substrate contained diaminobenzidine. Nuclei were counterstained with Carazzi hematoxylin. After immunostaining, tissue sections were examined under a light microscope (Zeiss). Using a computerized Microm image analyzer, percentages of elastin, LOX and LOXL in the entire thickness of the arterial wall were measured in 5 - $\mu \mathrm{m}$-thick cross-sections (20/study group). Percentages were compared among the study groups using the Mann-Whitney $U$ test. The level of significance was set at $\mathrm{p}<0.05$.

\section{Collagen Content}

Sirius red was used to localize and assess collagen types I and III in the arterial wall. This technique is based on the orientation and interaction between the sulfone groups of the dye and the amine groups of lysin and hydrolysin and guanidine groups of arginine in the collagen fibers, giving rise to different colors depending on the type of collagen. Collagen type I appears reddishorangey stained while type III collagen takes on a yellow-greenish shade. Using a computerized Microm image analyzer, percentages of collagens I and III were measured on 5 - $\mu$ m-thick crosssections (20/study group). The Mann-Whitney U test was used to compare these percentages among the different study groups. The level of significance was set at $\mathrm{p}<0.05$.

Lysyl Oxidases in Neointima

Development

\section{Results}

Macroscopic Observations

No signs of infection or acute graft rejection were observed in any of the recipient animals during the postoperative period.

In the fresh allograft group, 2 animals (7\%) died before the end of the study period (at 7 days and 2 months after implantation); however, it was not possible to establish the cause of death. Two of the grafts showed evident signs of thrombosis at 14 and 30 days after implantation. The patency rate was $93 \%$ for the fresh allografts.

\section{Morphological Observations}

A neointima of variable thickness could be seen to extend along the entire length of the graft (fig. 2a), including the suture zones and host tissue at the extreme ends, already at 14 days after implantation. The medial layer preserved its structure and characteristic cell population although the elastic laminae lost their even appearance to show irregular margins, degraded zones and extensive fenestrations. One month after grafting, the neointimal layer had thickened (fig. 2b). In some of these allografts, areas close to the anastomosis sites showed cell loss in the innermost third of the media. At 90 days, the neointimal layer had greatly increased its thickness by about two thirds with respect to 14 and 30 days. Thickening occurred at the expense of extracellular matrix synthesis more than cell proliferation.

The media of the fresh allografts showed evident signs of degeneration, with extensive cell loss along the entire graft length. One hundred and eighty-one days after surgery, neointimal thickening stabilized in the fresh allografts (fig. 2c, d). The medial layer, which was considerably degraded and clearly diminished in thickness, was acellular and supported by the discontinuous elastic laminae (fig. 2d).

In the group of animals treated with the LOX inhibitor for 30 days, we observed a thin neointimal layer compared to its appearance in the allografts not treated with the inhibitor. The selected study time was 30 days because at this time, intimal hyperplasia was consolidated in the nontreated group. In some areas, this thin neointimal layer was made up of 3 or 4 disorganized cell layers (fig. 2e, f). The structure and characteristic cell population of the medial layer were preserved. These observations indicate inhibition of intimal hyperplasia by the LOX inhibitor in the allografts, but no effects on the structure and characteristics of the preexisting extracellular matrix of the medial layer. 
Fig. 2. Light microscopic images of the arterial wall. $\mathrm{L}=$ Arterial lumen; $\mathrm{NI}=$ neointima; $\mathrm{M}=$ media; $\mathrm{A}=$ adventitia. a Arterial wall of a fresh allograft 14 days after implantation. Masson's trichrome. $\times 100$. b Graft 30 days after implantation showing increased thickening of the neointimal layer. Orcein. $\times 630$. c Arterial wall of an allograft 180 days after implantation. Orcein. $\times 100$. d Arterial wall 180 days after implantation, showing cell loss in the tunica media with evident signs of degeneration. Orcein. $\times 630$. e Arterial wall of an allograft 30 days after implantation, treated with the LOX inhibitor. Masson's trichrome. $\times 100$. $\mathbf{f}$ Arterial wall of an allograft image of the same group as in e, showing absence of intimal hyperplasia. Orcein. $\times 630$.

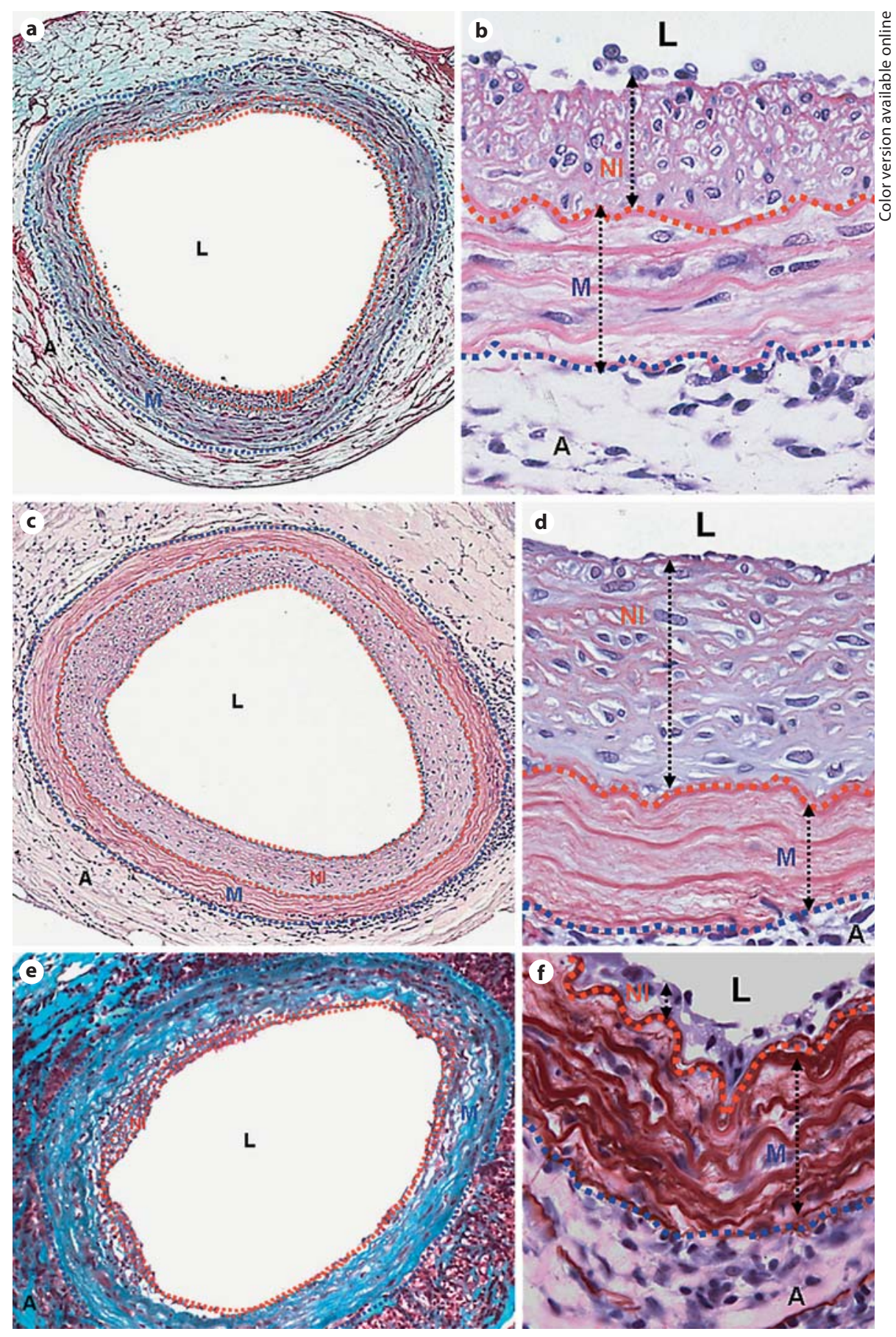

\section{Morphometry of Intimal Hyperplasia and the Tunica Media}

As early as 14 days after implantation, the arterial wall of the grafts was significantly thicker than in control specimens due to the presence of the neointima (fig. 3a). Differences in thickness between the short-term (14/30 days) and long-term (90/180 days) groups achieved significance at both study times, when the neointima reached its greatest thickness in the fresh allografts at 90 and 180 days (fig. 3a). This maximal hypertrophy started to decline at 6 months when the stenotic process became evident. In the group treated with the LOX inhibitor, neo- 

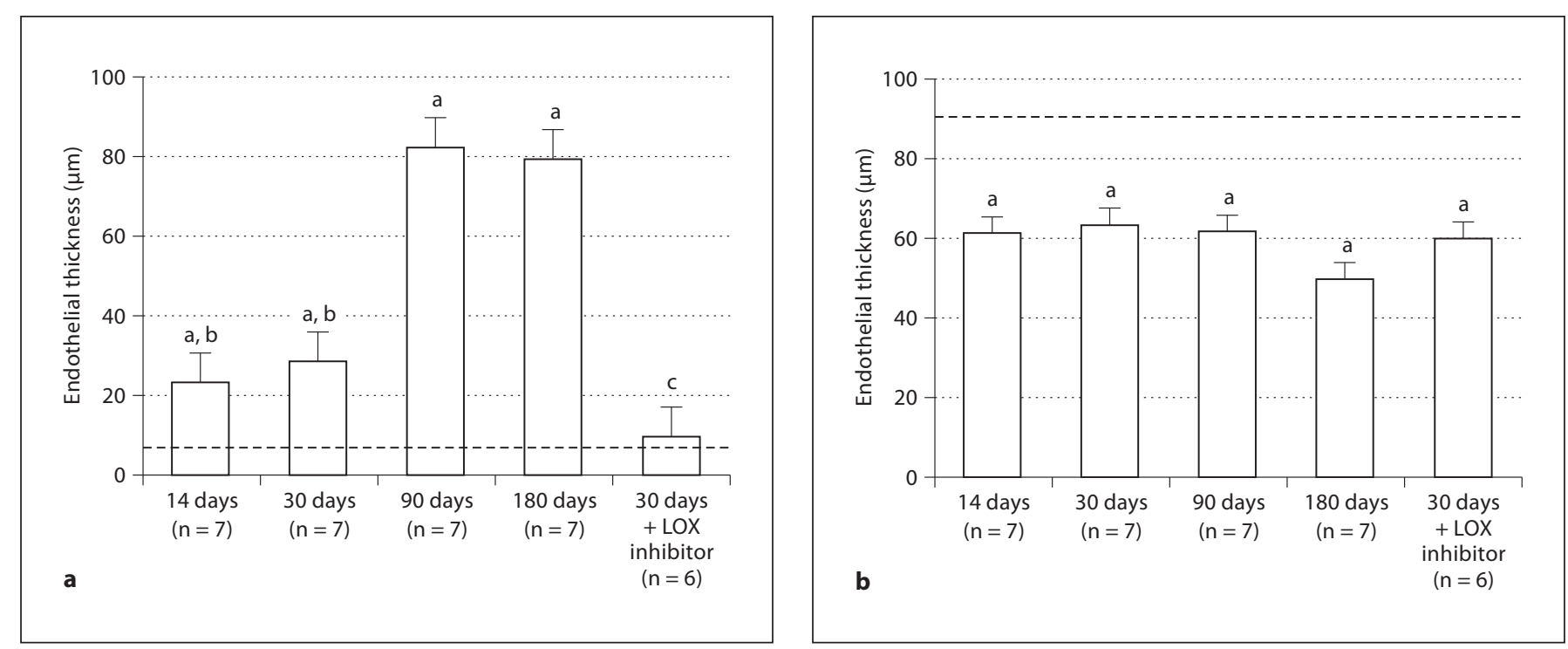

Fig. 3. a Morphometry of intimal hyperplasia in the different study groups. Dashed line = average endothelial thickness in control iliac arteries. ${ }^{a} \mathrm{p}<0.05$, with respect to the control iliac artery; ${ }^{\mathrm{b}} \mathrm{p}<0.05$ versus grafts of 90/180 days; ${ }^{c} \mathrm{p}<0.05$ versus 30/90/180 days. b Morphometry of the medial layer in the different study groups. Dashed line $=$ average medial thickness in control iliac arteries. ${ }^{a} \mathrm{p}<0.05$, with respect to the media of the control iliac artery.

intimal thickness was similar to that observed in the control group. Significant differences emerged when comparing the treated group with the rest of the groups at 30, 90 and 180 days (fig. 3a).

The thickness of the media was statistically significantly reduced at all the study times with respect to the control iliac artery (fig. 3b), even in the group treated with the LOX inhibitor. The involuted media showed its greatest reduction in thickness at 180 days, though the difference compared to the rest of the groups was not significant (fig. 3b).

\section{Immunohistochemical Analysis}

Tropoelastin

Tropoelastin (TE) expression was greatest at 14 days, and was observed throughout the thickness of the myointimal layer (fig. 4a), thereafter decreasing at 30 days and increasing in the medial layer at 90 and 180 days (fig. 4b). At 180 days, staining for TE was confined to the extracellular matrix and areas close to the elastic laminae of the medial layer, which had lost all its cells. Scarce staining was detected in the neointimal layer at 180 days. In the group treated with the LOX inhibitor, slight TE expression was confined to the intimal and adventitial layers (fig. 4c).

Lysyl Oxidases in Neointima Development
Percent TE expression was significantly greater at 30 days than at 14 days (table 1 ).

\section{LOX/LOXL1}

The expression patterns shown by LOX/LOXL1 were similar in all the grafts. At 14 days, labeling was intense in the fresh allografts and confined to the neointima, uppermost zones of the media and a few cells of the adventitia (fig. 4d, g).

At 30 days, cells scoring positive for LOX/LOXL1 in the grafts were observed in the neointima, media and adventitia. At 90 days, labeling persisted throughout the full thickness of the neointima and adventitia but was considerably reduced in the medial layer (fig. 4e). This loss was concurrent with the disappearance of the cell component. At 180 days, labeling for LOX was also detected throughout the neointima, though LOXL expression was mainly detected in the endothelium, and surface and deep zones of the adventitia (fig. $4 \mathrm{~h}$ ). In the group treated with the LOX inhibitor, LOX/LOXL expression was discrete in the intima and restricted to small areas of the media (fig. $4 \mathrm{f}$, i).

LOX and LOXL expression in the tissue sections differed significantly between the control artery and the short-term grafts (14/30 days, including the treated

J Vasc Res 2011;48:43-5 

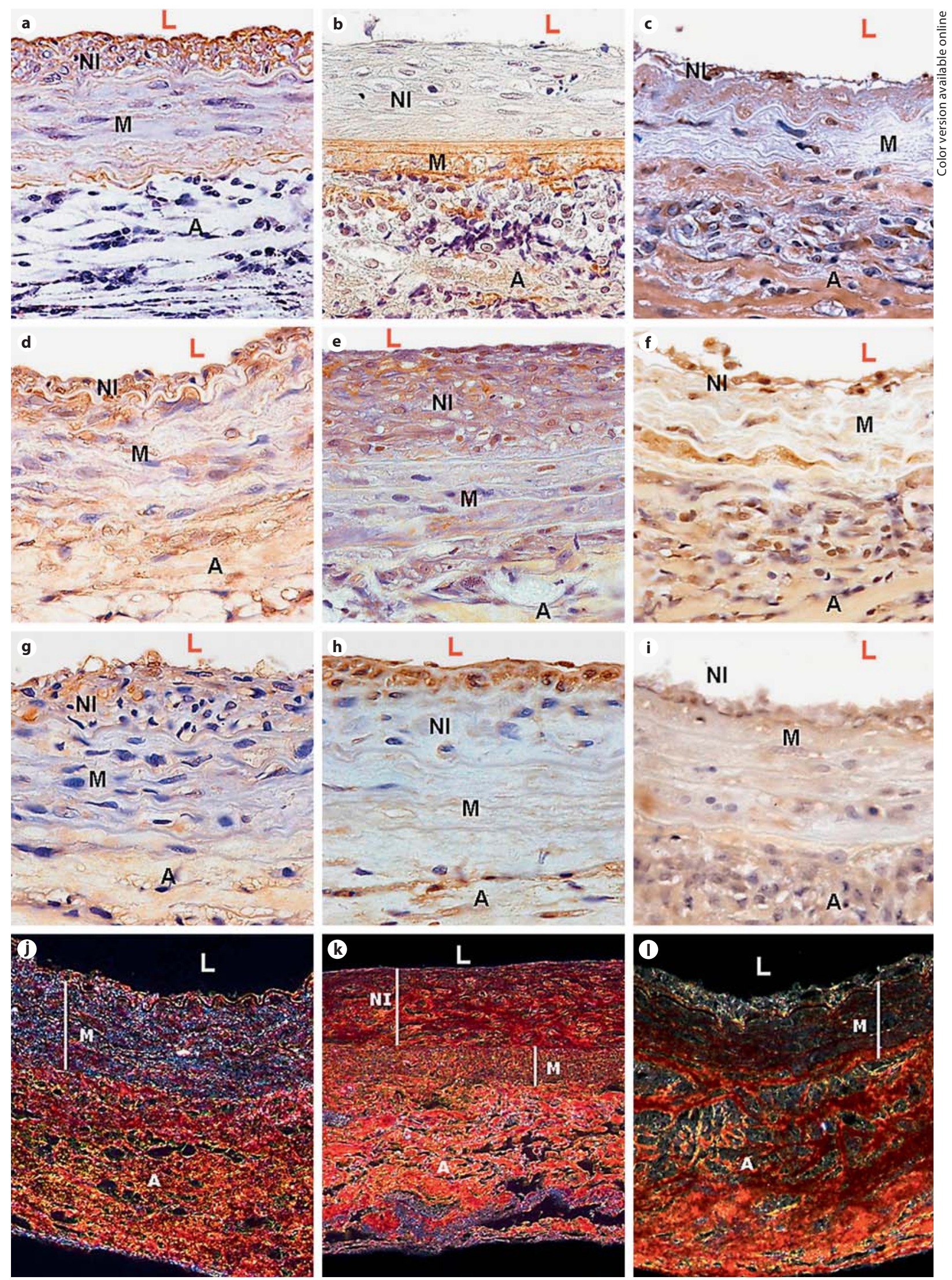
Table 1. Percentages of TE, LOX, LOXL, collagen I and collagen III recorded in the nonimplanted arterial segments (control artery) and in the allografts $14,30,90,180$ and 30 days + LOX after implant (mean \pm SD)

\begin{tabular}{|c|c|c|c|c|c|c|}
\hline \multirow[t]{2}{*}{ Staining for } & \multirow{2}{*}{$\begin{array}{l}\text { Control } \\
\text { artery } \\
(\mathrm{n}=5)\end{array}$} & \multicolumn{5}{|l|}{ Allograft } \\
\hline & & $\begin{array}{l}14 \text { days } \\
(\mathrm{n}=7)\end{array}$ & $\begin{array}{l}30 \text { days } \\
(n=7)\end{array}$ & $\begin{array}{l}90 \text { days } \\
(\mathrm{n}=7)\end{array}$ & $\begin{array}{l}180 \text { days } \\
(\mathrm{n}=7)\end{array}$ & $\begin{array}{l}30 \text { days + LOX } \\
(n=6)\end{array}$ \\
\hline $\mathrm{TE}$ & $12.9 \pm 6.1$ & $9.5 \pm 2.1$ & $20.3 \pm 4.5^{*}$ & $15.1 \pm 3.7$ & $12.4 \pm 4.4$ & $13.6 \pm 5.2$ \\
\hline LOX & $6.6 \pm 2.9$ & $10.6 \pm 4.2$ & $11.1 \pm 2.5$ & $28.2 \pm 2.7^{*}$ & $26.6 \pm 5.8^{*}$ & $8.2 \pm 5.6$ \\
\hline LOXL & $4.2 \pm 4.7$ & $6.8 \pm 2.9$ & $9.2 \pm 4.3$ & $20.1 \pm 6.0^{*}$ & $18.2 \pm 2.1^{*}$ & $8.6 \pm 2.4$ \\
\hline Collagen I & $31.4 \pm 6.2$ & $38.1 \pm 8.3$ & $41.3 \pm 7.6$ & $50.8 \pm 10.4^{* *}$ & $65.2 \pm 8.4^{*}$ & $27.8 \pm 4.6$ \\
\hline Collagen III & $10.3 \pm 3.3$ & $25.4 \pm 7.3^{*}$ & $22.8 \pm 5.7^{*}$ & $12.8 \pm 5.2$ & $9.5 \pm 4.1$ & $13.3 \pm 8.1$ \\
\hline
\end{tabular}

TE: ${ }^{*} \mathrm{p}<0.05$, vs. 14 days; LOX: ${ }^{*} \mathrm{p}<0.05$, vs. the rest of the groups; LOXL: ${ }^{*} \mathrm{p}<0.05$, vs. the rest of the groups; collagen I: ${ }^{*} \mathrm{p}<$ 0.05 , vs. the rest of the groups, ${ }^{* *} \mathrm{p}<0.05$, vs. control and $30+\mathrm{LOX}$; collagen III: ${ }^{*} \mathrm{p}<0.05$, vs. control artery and 180 days.

group) with respect to long-term (90/180 days) grafts (table 1).

\section{Collagen Content}

In control arterial segments, collagen was mainly of type I and, as expected, confined to the adventitial layer. Fourteen days after implantation, the grafts showed the two forms of collagen (fig. $4 \mathrm{j}$ ), observed as a reddening in the color of the neointima over time. At 30 days, the neointima showed a greater amount of collagen III, which subsequently diminished at the expense of increasing collagen I, such that at 180 days, most collagen was type I (fig. 4k). In the animals treated with the LOX inhibitor, collagen was mainly of type I and appeared in the medial and adventitia layers. The thin intimal layer showed scarce collagen expression (fig. 4l).

Fig. 4. Immunohistochemical staining for tropoelastin in arterial allograft walls 14 days after implantation (a), 90 days after implantation (b), and 30 days after implantation + LOX inhibitor (c). $\times 630$. Immunohistochemical staining for LOX in arterial allograft walls 14 days after implantation (d), 90 days after implantation (e), and 30 days after implantation + LOX inhibitor (f). $\times 630$. Immunohistochemical staining for LOXL in arterial allograft walls 14 days after implantation (g) 180 days after implantation (h), and 30 days after implantation + LOX inhibitor (i). $\times 630$. Sirius red staining for collagen III (yellow) and collagen I (red) in arterial allograft walls 14 days after implantation (j), 180 days after implantation (k), and 30 days after implantation + LOX inhibitor (I). $\times 630 . \mathrm{L}=$ Arterial lumen; $\mathrm{NI}=$ neointima; $\mathrm{M}=\mathrm{me}$ dia; $\mathrm{A}=$ adventitia.

Lysyl Oxidases in Neointima Development
Collagen I expression in the graft walls was significantly greater at 180 days after implantation compared with the rest of the groups and at 90 days with respect to control arteries/30 days + LOX inhibitor (table 1). On the contrary, collagen III underwent a significant drop in the long-term (180 days) grafts relative to the short-term ones (14/30 days) (table 1$)$.

\section{Discussion}

The extracellular matrix is a complex dynamic environment with both cell and fibrillar components that is able to accommodate changes in volume or density; it also stores and releases signaling molecules, such as growth factors and/or cytokines. The extracellular matrix has its own mechanisms for the regulation, renewal and repair of blood vessels after injury, including the development of intimal hyperplasia $[9,10]$.

Intimal hyperplasia starts off as a layer that emerges from beneath the endothelium. The thickness of this layer increases from the initial moments of grafting and in our study, its thickness stabilized at 3 months after implantation. This layer, which arises as a consequence of the aggression suffered by the wall, forms part of the wound repair process that follows any injury and is attributed to the arterial complications that occur between the first 2 months and 2 years of vascular surgery.

In our model of fresh allografts, we observed that the graft develops a neointima of variable thickness as early as 14 days after surgery. This neointima increased in thickness until 90/180 days after implantation, when cell loss and the consequent degradation of the medial layer 
took place. This last process cannot be ascribed to the immune response since it has been reported to similarly occur in a syngeneic transplant model [11]. It is likely that the grafting process induces cell or perhaps molecular changes in the extracellular matrix that impair the onset of the proliferative and migratory response. We differ from authors who attribute intimal hyperplasia and other vessel wall alterations not observed in isogenic grafts, such as medial cell loss, to the host immune response [12, 13]. In syngeneic and allogeneic grafts conducted by our group using the same model, we have always observed a similar behavior. Thus, sooner or later after surgery, the grafted arterial segments are transformed into elastic tubes that preserve their function due to the neointimal layer.

As key enzymes stabilizing collagen and elastin, LOX will be directly involved in the extracellular matrix deposition that occurs when a neointima develops. In effect, we observed increased expression of these enzymes during the whole growth process of the neointima as well as in later stages, suggesting they could be an important factor for the appearance of stenotic lesions. If the activity of these enzymes could be controlled in some way, the development of this type of lesion could perhaps be modulated. LOX enzyme inhibitors such as BAPN have been reported to modulate vessel lumen narrowing in a rabbit balloon angioplasty model [14]. Other authors using LOX inhibitors have reported, in a model of Brown Norway rats, decreased carotid wall rupture, decreased aortic insoluble elastin and increased defects in the internal elastic lamina of different arteries [15].

In our model, treatment with inhibitors of LOX activity for 30 days prevented the development of intimal hyperplasia after the implantation of a vascular substitute. In this model, LOX and LOXL expression increases as intimal hyperplasia progresses from 14 to 90/180 days, when the neointimal layer was at its highest thickness and collagen I expression.

No previous study has examined LOX expression after the implantation of an arterial allograft. In effect, only a few investigations have explored the expression of LOX in models of vascular injury. In an experimental model of balloon catheter-induced vascular damage, 3 days after surgery, Nuthakki et al. [16] observed increased LOX expression that persisted until 21 days after surgery and returned to normal levels at 28 days, when the greatest increases in intimal thickness and collagen accumulation were observed. Nikkari et al. [17] observed significantly elevated expression of versican, biglycan, collagen, and elastin mRNA 1-2 weeks after balloon catheter injury in the rat carotid artery. In addition, a role of LOX in cardiovascular function has been indicated by a high incidence of aortic aneurysms, aortic tortuosity, and signs of aortic rupture in LOX-/- knockout mice [3].

We observed enhanced expression of type I collagen at 30 days after implantation, which rose with time both in the adventitia and neointima. A further observation was intense labeling in the neointima at the 180-day time point. This finding indicates that during intimal growth, collagen I synthesis takes place, involving the inevitable actions of LOX in collagen cross-linking as reflected by their immunohistochemical localization. Similar results have been reported by Nili et al. and Strauss et al. [18, 19] in balloon catheter-induced injury models, in which, through biochemical techniques, a significant increase in the amounts of both collagen and elastin was detected 7-60 days after surgical insult to the rat carotid artery. This demonstrates that extracellular matrix formation is essential for the development of restenotic lesions and suggests that approaches designed to promote or expedite either the decreased synthesis or increased turnover of these extracellular matrix components could affect restenosis independently of cell proliferation. Other authors $[17,20]$ have noted elevated TE, type I collagen and proteoglycan mRNA levels as early as 1 week after injury, with gene expression persisting until 2-3 weeks after injury. In a pig model of interposition grafting of saphenous vein into carotid artery [21], the authors described steady thickening of the tunica intima and media from 2 to 168 days after surgery and increased production of matrix metalloproteinases (MMP)-2 and -9, two basement membrane-degrading enzymes, during neointima formation. These authors proposed MMPs as new therapeutic targets for preventing late graft failure.

In summary, the most significant findings of our study were the overexpression of LOX during late stages of intimal hyperplasia and its prevention using a LOX inhibitor. Modulation of LOX activity could thus be a feasible option for avoiding excessive extracellular matrix deposition and consequent stenosis in small-caliber vessels.

\section{Acknowledgments}

We thank Dr. Robert P. Mecham for providing the anti-tropoelastin antibody and Dr. Sommer for the anti-LOX/LOXL1 antibodies. This study was supported by a grant from the Fondo de Investigación Sanitaria (FIS-PI 06/1781). 


\section{References}

1 Platt JL: Immunobiology of xenotransplantation. Transpl Int 2000;13:7-10.

$\checkmark 2$ Bordenave L, Menu P, Baquey C: Developments towards tissue-engineered, small-diameter arterial substitutes. Expert Rev Med Devices 2008;5:337-347.

>3 Rodríguez C, Martínez-González J, Raposo B, Alcudia JF, Guadall A, Badimon L: Regulation of lysyl oxidase in vascular cells: lysyl oxidase as a new player in cardiovascular diseases. Cardiovasc Res 2008;79:7-13.

$\checkmark 4$ Rodríguez C, Raposo B, Martínez-González J, Casani L, Badimon L: Low density lipoproteins downregulate lysyl oxidase in vascular endothelial cells and the arterial wall. Arterioscler Thromb Vasc Biol 2002;22:1409_ 1414.

5 Maki JM, Rasanen J, Tikkanen H, Sormunen R, Mäkikallio K, Kivirikko KI, Soininen R: Inactivation of the lysyl oxidase gene Lox leads to aortic aneurysms, cardiovascular dysfunction, and perinatal death in mice. Circulation 2002;106:2503-2509.

-6 Pinnell SR: Molecular defects in the EhlersDanlos syndrome. J Invest Dermatol. 1982; 79:90-92.

-7 Kuivaniemi H, Peltonen L, Kivirikko KI: Type IX Ehlers-Danlos syndrome and Menkes syndrome: the decrease in lysyl oxidase activity is associated with a corresponding deficiency in the enzyme protein. Am J Hum Genet. 1985;37:798-808.
8 Khakoo A, Thomas R, Trompeter R, Duffy P, Price R, Pope FM: Congenital cutis laxa and lysyl oxidase deficiency. Clin Genet. 1997;51: 109-114.

99 Galis ZS, Khatri JJ: Matrix metalloproteinases in vascular remodelling and atherogenesis: the good, the bad and the ugly. Circ Res 2002;90:251-262.

10 Kuzuya M, Kanda S, Sasaki T, Tamaya-Mori $\mathrm{N}$, Cheng XW, Itoh $\mathrm{T}$, Itohara S, Iguchi A Deficiency of gelatinase A suppresses smooth muscle cell invasion and development of experimental intimal hyperplasia. Circulation 2003;108:1375-1381.

- 11 Pascual G, Martínez S, Rodríguez M, Serrano $\mathrm{N}$, Bellón JM, Buján J: Patency and structural changes in cryopreserved arterial grafts used as vessel substitutes in the rat. J Surg Res 2005;124:297-304.

12 Hirsch GM, Kearsey J, Burt T, Karnovsky MJ, Lee T: Medial smooth muscle loss in ar terial allografts occurs by cytolytic cell induced apoptosis. Eur J Cardiothorac Surg 1998;14:89-97.

13 Bojakowski K, Religa P, Bojakowska M, Hedin U, Gaciong Z, Thyberg J: Arteriosclerosis in rat aortic allografts: early changes in endothelial integrity and smooth muscle phenotype. Transplantation 2000;70:65-72.

14 Spears JR, Zhan H, Khurana S, Karvonen RL, Reiser KM: Modulation by $\beta$-aminopropionitrile of vessel luminal narrowing and structural abnormalities in arterial wall collagen in a rabbit model of conventional balloon angioplasty versus laser balloon angioplasty. J Clin Invest 1994;93:1543-1553.
15 Mercier N, Kakou A, Challande P, Lacolley P, Osborne-Pellegrin M: Comparison of the effects of semicarbazide and $\beta$-aminopropionitrile on the arterial extracellular matrix in the Brown Norway rat. Toxicol Appl Pharmacol 2009;239:258-267.

$>16$ Nuthakki VK, Fleser PS, Malinzak LE, Seymour ML, Callaham RE, Bendick PJ, Zelenock GB, Shanley CJ: Lysyl oxidase expression in a rat model of arterial ballon injury. J Vasc Surg 2004;40:123-129.

17 Nikkari ST, Jarvelainen HT, Wight TN, Ferguson M, Clowes AW: Smooth muscle cell expression of extracellular matrix genes after arterial injury. Am J Pathol 1994;144: 1348-1356.

18 Nili N, Zhang M, Strauss BH, Bendeck MP: Biochemical analysis of collagen and elastin stynthesis in the balloon injured rat carotid artery. Cardiovasc Pathol 2002;11:272-276.

19 Strauss BH, Chisholm RJ, Keeley FW, Gotlieb AI, Logan RA, Armstrong PW: Extracellular matrix remodeling after balloon angioplasty injury in a rabbit model of restenosis. Circ Res 1994;75:650-658.

20 Majesky MW, Giachelli CM, Reidy MA, Schwartz SM: Rat carotid neointimal smooth muscle cells reexpress a developmentally regulated mRNA phenotype during repair of arterial injury. Circ Res 1992;71:759-768.

-21 Southgate KM, Mehta D, Izzat MB, Newby AC, Angelini GD: Increased secretion of basement membrane degrading metalloproteinases in pig saphenous vein into carotid artery interposition grafts. Arterioscler Thromb Vasc Biol 1999;19:1640-1649. 